\title{
Fundus Image Classification Using Convolutional Neural Network
}

\author{
U.Savitha $^{\text {a }}$, Kodali Lahari Chandana ${ }^{\text {a,1 }}$, A.Cathrin Sagayam ${ }^{\text {a, }}$ S. Bhuvaneswari ${ }^{\text {a }}$ \\ ${ }^{a}$ Department of Electronics and Communication Engineering, \\ Vel Tech HighTech Dr Rangarajan Dr Sakunthala Engineering College, Chennai, TN, \\ India
}

\begin{abstract}
Different eye disease has clinical use in defining of the actual status of eye, in the outcome of the medication and other alternatives in the curative phase. Mainly simplicity, clinical nature are the most important requirements for any classification system. In the existing they used different machine learning techniques to detect only single disease. Whereas deep learning system, which is named as Convolutional neural networks (CNNs) can show hierarchical representing of images between disease eye and normal eye pattern.
\end{abstract}

Keywords. Fundus image disease, glaucoma, Macular edema, Eye lesion, machine learning, Deep learning technique, image processing.

\section{Introduction}

The Eye, it is the visual organ and the most important of the special senses. We know the situation is very horrible when we don't have sight. Blindness knows no geographic, economic, cultural bonds. It will have no concern for age or gender [1]. Blindness cases are progressing on a daily basis. The NPCB was appeared in 1976, to reduce prevalence of blindness from 1.4 to $0.3 \%$. The vision 2020 "The Right to Sight" may be a worldwide scheme to scale back averted blindness [2]. They mainly targeted cataract, refractive error childhood blindness, corneal blindness, glaucoma is shown in Figure 1. Some eye problems may be small, but some other may lead to loss of vision [3-6]. Common eye problems which were mainly refractive errors, cataracts, optic nerve disorders, Glaucoma. Retinal disorders etc. Mainly eye diseases were due to use of modern medicines and treatment methods, this was noted by Edward Kondort MD, $\mathrm{CCH}$, DHt (human homeopathic ophthalmologist)

1.1 Macular Edema: It is the build from fluid within the macula, which is that the area within the centre of the retina. The retina is present at the rear of the attention which is light sensitive tissue and macula is that the a segment of the Retina, which is liable for precise and straight-forward vision. The macula stretches and elongates as a result of the fluid buildup, in that we would lose our sight. 
1.2 Glaucoma: It is disease that damages the eye's nervous optics. It happens when fluid builds up ahead a part of the attention. This fluid buildup a pressure, which is understood as intraocular pressure (IOP), is high. Increase within the vital sign which causes the loss of blindness. it's a grave disease and each case that's untreated ends in blindness.

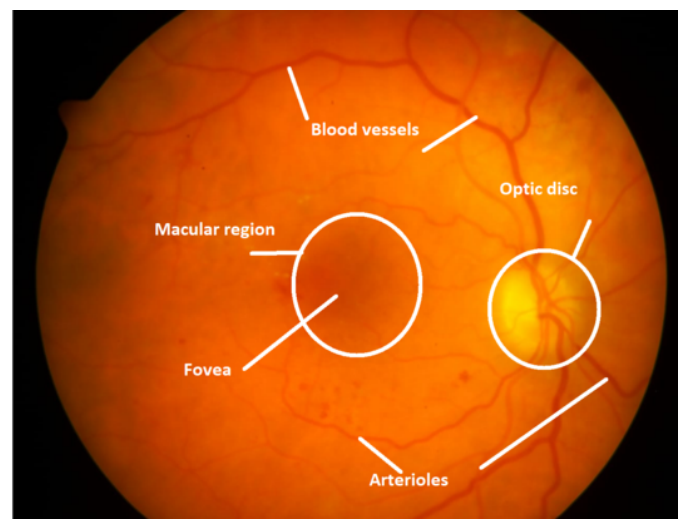

Figure 1. Eye glaucoma

1.3 Eye lesion: Begin lesion have several characteristics that differ them from malignant lesions, a number of the kinds begins which include squamous papilloma, keratoacanthoma, hereditary begin intraepithelial dyskeratosis etc. It is often treated by surgical excision, cryotherapy or topical chemotherapy.

\section{Existing System}

Diabetes may be a disease during which, an individual fails to respond to insulin released by their pancreas, or pancreas doesn't produce sufficient insulin [7]. Early detection of diabetic disease is completed through advanced machine learning techniques over manual detection. This paper presents a scientific survey of automated approaches to diabetic disease from several aspects, which are named as Available data sets, image preprocessing techniques, deep learning models and performance evaluation metrics. The synopsis of diabetic disease detection is given by our survey.

\section{ADVANTAGES:}

1) Fast treatment results.

2) Accurate results.

\section{DISADVANTAGES:}

1) It detects only analysis of a diabetic disease.

\section{Proposed System}

In Our paper, we presented a deep learning technique designed to detect the disease. In this proposed system, deep learning model is implemented to detect various eye diseases like macular edema, glaucoma and eye lesions [8]. One of the foremost famous deep learning algorithms most commonly utilized in image classification is a 
convolutional neural network. In general, this consists of three layers namely convolutional layer, pooling layer, and fully connected layer is shown in Figure 2. The CNN design picks up an image as input, which is then carried via the layers to detect features and identify the image, and yielding a response.

\section{ADVANTAGES:}

Here we classify different disease like macular edema, eye lesion and glaucoma detection.

1) Highly reliable.

2) Fast treatment results.

3) Accurate results.

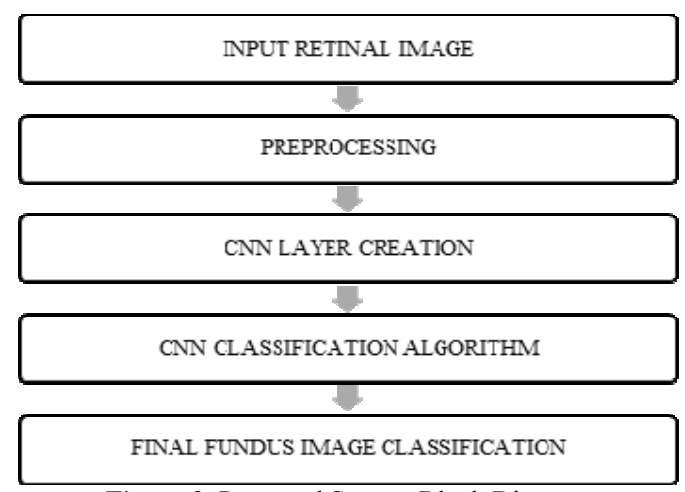

Figure 2. Proposed System Block Diagarm

\subsubsection{Input Retinal Image:}

We begin by downloading the images into MATLAB. Dataset's area unit holds on in many alternative file varieties. This image is stored as binary files that MATLAB will quickly use and reshape into pictures.

\subsubsection{Preprocessing:}

Data pre-processing may even be a method of making ready the information and creating it appropriate for a machine learning model [9-16]. The data typically consists of noises, mission values, maybe unusable formats that can't be used directly for machine learning models. Pre-processing is used to cleansing the information and creating it appropriate for a machine learning model. This will increase the accuracy and potency of the machine learning models.

\subsubsection{CNN Layer Creation:}

The Convolutional Neural Networks $(\mathrm{CNN})$ is one in all the foremost notable deep learning algorithms and therefore, this was ordinarily utilized in image classification applications. This algorithmic software takes an input image and runs it via the levels to classify choices and recognize the image, resulting in a classification outcome. This design usually involves alternating convolutional layers and pooling layers, after that it follows by a slew of fully connected layers. At regular intervals, the output is the input of the next layer. In Figure 3, the CNN input is also a three-dimensional image (width $\times$ height $\times$ depth), the breadth, peak area unit is the size of the images. The depth is the number of input nodes, which are three color channels Red, Green, and Blue (RGB). 
This layer brings out options via pictures. Every convolutional has weighted matrices and the area unit referred to as filters or kernels that float across the input part of image to explicit data. The CNN's first layers look for colors and simple patterns. Thus, at intervals the next layers step by step find a lot of complicated patterns. To generate a function map, each filter performs a convolution process.
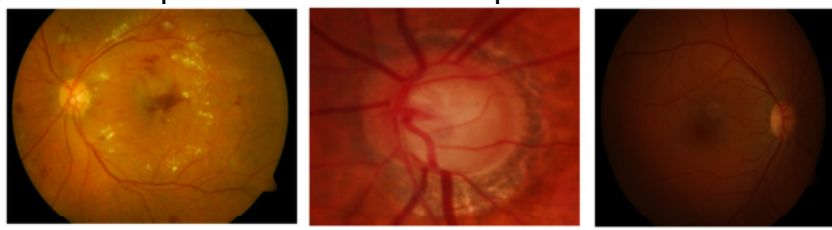

Figure 3. Proposed CNN Layer Creation

\section{Equations}

We have a picture " $x$ ", which may be a $2 \mathrm{D}$ array of pixels with different color channels (Red, Green and Blue-RGB) and that we have a feature detector or kernel "w" then the output we get after applying a mathematical process is named a feature map.

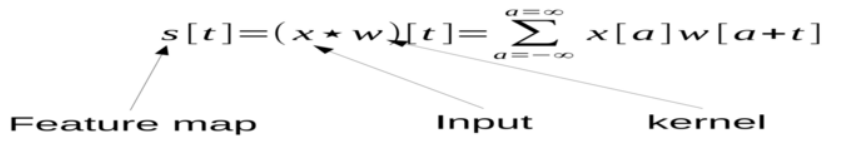

This process aids in determining the correlation between two signals. The feature detector will recognize edges within the images. Thus, the operation can assist us in identifying the sides within the image until such a filter is applied to the image. These functions are usually assumed to be zero everywhere but their values are stored at a finite number of points. Thus, this helps us to implement a sum of a given range of array element.

$$
S(i, j)=(I * K)(i, j)=\sum_{m} \sum_{n} I(m, n) K(i-m, j-n)
$$

Where I is two dimensional array is Kernel function The above equation is rewritten as cross correlation function, since the convolution function is independent

$$
S(i, j)=(K * I)(i, j)=\sum_{m} \sum_{n} I(i-m, j-n) K(m, n)
$$

For Example ,if we saw a person or an image in a dark how could we identify the image? We would just turn on our flashlight and sacn across the image. That is why we neutralize convolutional layer. 


\section{Results}
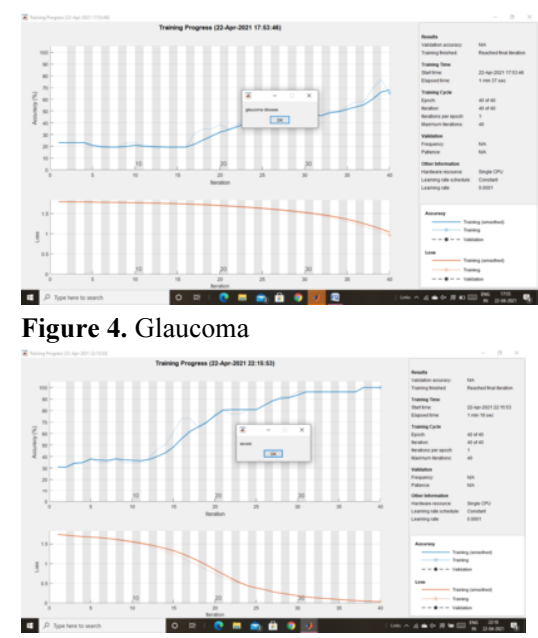

Figure 6. Macular Edema

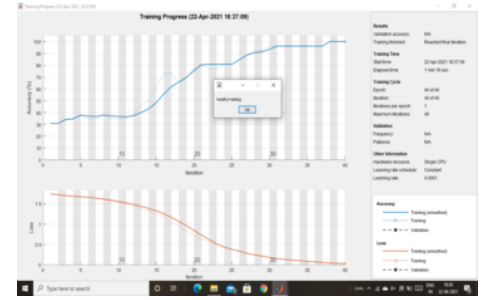

Figure 5. Healthy Eye

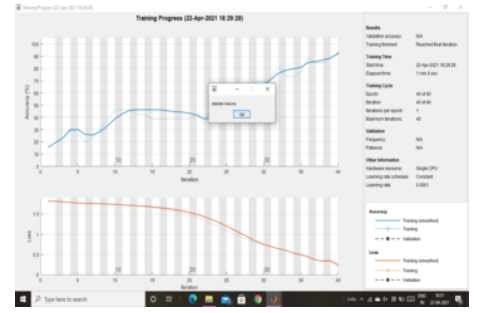

Figure 7. Eye Lesion

Eye lesion: In the above Figure 7 results, eye lesion can be found out by seeing the pop-up message as diabetic lesion. Accuracy obtained $92 \%$ and the loss is 0.25 .

Macular Edema: In the above Figure 6 results, macular edema can be found out by seeing the pop-up message as severe.

Accuracy obtained $100 \%$ and the loss is 0 .

Glaucoma: In the above Figure 4 results, glaucoma can be found out by seeing the popup message as glaucoma disease.

Accuracy obtained $69 \%$ and the loss is 1.1 .

Healthy Eye: In the above Figure 5 results, healthy eye can be found out by seeing the pop up message as healthy stating.

Accuracy obtained $100 \%$ and the loss is 0 .

\section{Conclusion}

In our proposed system we have implemented a method for the classification of diabetic eye diseases using Convolutional Neural Network. Our method primarily identifies diseases such as macular edema, glaucoma, eye lesion and healthy eye. We classify the images as regular, extreme or abnormal based on the measurements of exudates from the macula. We also tested many datasets, which gave us excellent results. Our proposed method, which is integrated into a real-time patient management system, will perform automated diagnoses on medical images for diabetic complications

\section{References}

[1] Park, K. (2015). Park's Textbook of Preventive and Social Medicine 23rd edition, page no.723. Bansaridas Bhanot, 404-13. Retrieved from http://www.parasredkart.com/index.php?route=product/ product\&product_id $=4544$

[2] Sandhar Singh Harbakhash. Textbook of Opthalmology and Otology with Homeopathic Therapeutics, 1st edition, New Delhi, B.Jain Publishers, 2003. 
[3] Todd Cooney. Homeopathy for eye disorders info (internet) (cited 9 feb 2017) available from: https://ivcjournal.com/homeopathy-eye-disorders

[4] Book Review: Handbook of Opthalmology. (1951). Canadian Journal of Occupational Therapy, 18(3), 95-95. https://doi.org/10.1177/000841745101800306

[5] Sathye, S. (2017). Effect of homoeopathic preparation of Ruta graveolens on the progression of childhood myopia before, during and after cessation of treatment: A retrospective study. Indian Journal of Research in Homoeopathy, 11(4), 249. https://doi.org/10.4103/ijrh.ijrh_72_16

[6] Verma. S.P. Practical Handbook of Surgery with Homeopathic Therapeutics, Reprinted edition, New Delhi, B. Jain Publishers(P)Ltd, 2007.

[7] Norton A.B. Opthalmic Diseases and their Homeopathic Therapeutics, 3rd edition, New Delhi, B.Jain Publishers (P) Ltd., 2005.

[8] Chand DH. Role of Homoeopathy in Opthalmological Conditions, Indian J Opthalmol, 1982, Volume: 30, Page 245- 248.

[9] A.Kunwar, S.Magotra, and M.P.Sarathi, "Detection of high -risk macular edema using texture features and classification using SVM classier," in proc. Int. Conf. Adv. Comput., Commun. Inform. (ICACCI), Aug. 2015, pp. 22852289

[10] S. J. J. Kumar and C. G. Ravichandran, "'Macular edema severity detection in colour fundus images based on ELM classier," in Proc. Int.Conf. I-SMAC (IoT Social, Mobile, Anal. Cloud) (I-SMAC), Feb. 2017, pp. 926933.

[11] Wu, W., Zhang, H., Pirbhulal, S., Mukhopadhyay, S. C., \& Zhang, Y. T. (2015). Assessment of Biofeedback Training for Emotion Management Through Wearable Textile Physiological Monitoring System. IEEE Sensors Journal, 15(12), 7087-7095. https://doi.org/10.1109/JSEN.2015.247063

[12] Pirbhulal, S., Zhang, H., Wu, W., Mukhopadhyay, S. C., \& Zhang, Y. T. (2018). Heartbeats Based Biometric Random Binary Sequences Generation to Secure Wireless Body Sensor Networks. IEEE Transactions on Biomedical Engineering, 65(12), 2751-2759. https://doi.org/10.1109/TBME.2018. 2815155

[13] Sodhro, A. H., Pirbhulal, S., \& Sangaiah, A. K. (2018). Convergence of IoT and product lifecycle management in medical health care. Future Generation Computer Systems, 86, 380-391. https://doi.org/10.1016/j.future.2018.03.052

[14] Wu, W., Pirbhulal, S., Sangaiah, A. K., Mukhopadhyay, S. C., \& Li, G. (2018). Optimization of signal quality over comfortability of textile electrodes for ECG monitoring in fog computing based medical applications. Future Generation Computer Systems, 86, 515-526. https://doi.org/10.1016/ j.future.2018.04.024

[15] Jamal, I., Akram, M. U., \& Tariq, A. (2012). Retinal Image Preprocessing: Background and Noise Segmentation. TELKOMNIKA (Telecommunication Computing Electronics and Control), 10(3), 537. https://doi.org/10.12928/telkomnika.v10i3.834 